\title{
Zuhause im Quartier
}

Der Paritätische Wohlfahrtsverband in Nordrhein-Westfalen setzt innovative Wohnmodelle für alle Generationen um. Denn zu Hause alt werden, das wünschen sich rund zwei Drittel der Seniorinnen und Senioren in Deutschland. Die Pflegestatistik zeigt, dass bundesweit 70 Prozent der pflegebedürftigen Menschen zu Hause versorgt werden - sie wollen im Alter in ihrer vertrauten Umgebung wohnen bleiben. Aber auch die jüngere Generation und Familien mit Kindern möchten sich in ihrem Stadtviertel wohl fühlen, soziale und nachbarschaftliche Netzwerke nutzen.

Bereits 2006 haben sich der Paritätische Nordrhein-Westfalen, der VdW Rheinland Westfalen und der Deutsche Mieterbund Nordrhein-Westfalen in der "Dortmunder Erklärung" (www.wohnen-im-alternrw.de) verpflichtet, nutzerfreundliche Wohnmodelle für ältere Menschen zu entwickeln und nachhaltig zu gestalten. Gemeinsam mit seinen Mitgliedsorganisationen und Kooperationspartnern setzt sich der Verband für eine quartiersnahe Versorgung ein. Generationsübergreifendes Wohnen im angestammten Quartier ist der Leitgedanke für diese innovativen Wohnformen. Unter dem Dach des Paritätischen versammeln sich derzeit rund 70 ambulant betreute Wohngemeinschaften sowie knapp 20 generationsübergreifende Wohn- und zahlreiche Quartiersprojekte.

Wenn ältere Menschen aufgrund einer Erkrankung oder Behinderung den Alltag nicht mehr alleine bewältigen können, benötigen sie niedrigschwellige Hilfsangebote innerhalb ihres Wohnviertels. Nachbarschaftliche Netzwerke, eine Haushaltshilfe und ambulante Pflegedienste unterstützen die Betroffenen, damit sie ein selbstständiges Leben führen können. Auch eine Wohnraumberatung hilft ihnen, ihre Wohnung barrierearm oder barrierefrei zu gestalten. Ist der

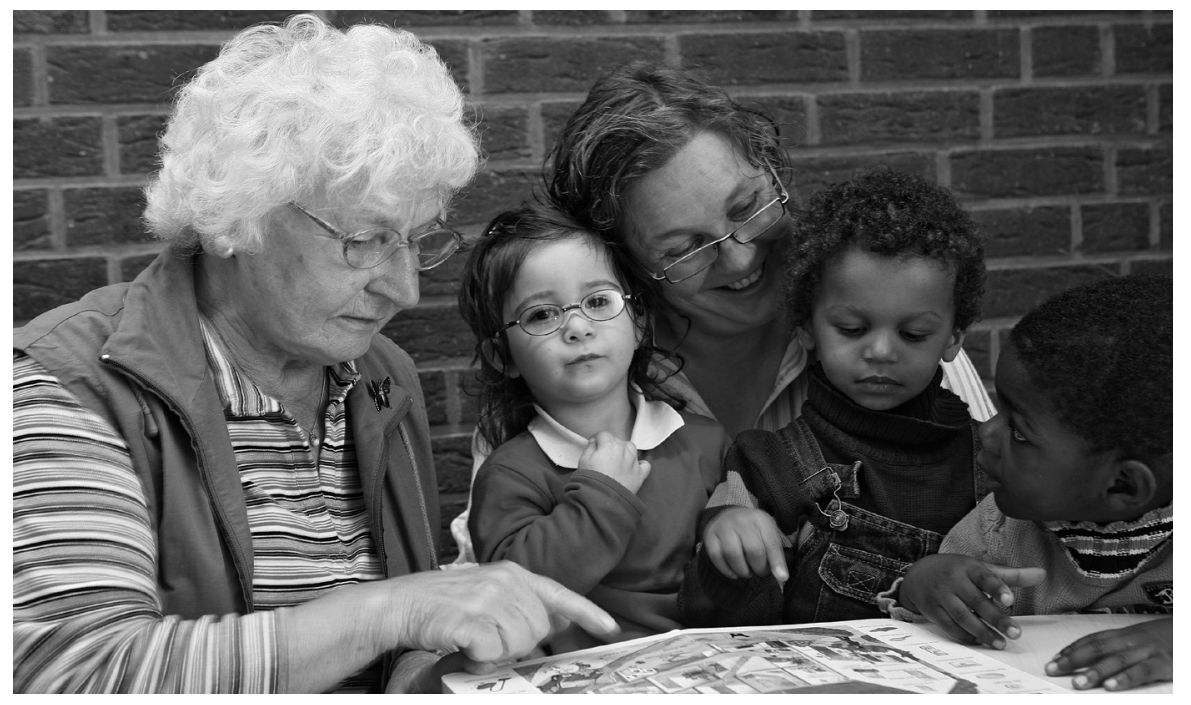

Verbleib in der eigenen Häuslichkeit nicht mehr möglich, kann der Umzug in eine ambulant betreute Wohngemeinschaft eine Alternative sein. Diese Versorgungsart lässt sich aufgrund ihrer überschaubaren Größe sehr gut ins Quartier integrieren und bietet den BewohnerInnen viele Vorteile - unter anderem werden sie dort rund um die Uhr betreut.

Generationsübergreifende Wohnprojekte, von denen es in Nordrhein-Westfalen noch deutlich zu wenige gibt, zeigen, wie man ein Quartier alters- und sozialdurchmischt entwickelt. Um ein Stadtviertel lebendig zu gestalten, müssen sich ältere und jüngere Menschen, Familien und Alleinerziehende mit Kindern dort wohl fühlen. Alle Generationen wünschen sich ein Umfeld, das geprägt ist von Aspekten wie Geborgenheit, Sicherheit, Vertrautheit, und Erreichbarkeit - ihr Zusammenleben erfordert jedoch auch Toleranz und Kompromissbereitschaft. Darüber hinaus sind Nachbarschaftsvereine ein wichtiges Bindeglied, um Kontakte innerhalb der Nachbarschaft zu pflegen: Sie schaffen Orte der
Begegnung und des Engagements mitten im Quartier.

Der Paritätische Wohlfahrtsverband verstärkt mit seinem Kompetenzzentrum "Wohnen im Alter NRW" und dem gleichnamigen Informationsportal im Internet seine Aktivitäten zur Quartiersentwicklung. In den vergangenen Jahren hat der Verband einen Zulauf an gemeinnützigen Vereinen verzeichnet, die im breiten Themenfeld "Wohnen im Alter" ihre Vorstellungen verwirklichen möchten. Dabei wird der Paritätische seine Mitglieder unterstützen und helfen, fehlende Angebote zu initiieren sowie Hindernisse aus dem Weg zu räumen. Auch die Landesregierung fördert strukturell und ressortübergreifend mit dem "Masterplan Quartier" die Umsetzung von Quartiersprojekten.

Franz Schumacher

Fachreferent "Wohnen im Alter" und "Ambulante Pflege « beim Paritätischen Wohlfahrtsverband, Landesverband NordrheinWestfalen e. V.

www.wohnen-im-alter-nrw.de
- Aufbau und Aktivierung einer tragenden sozialen Infrastruktur: So wie es keine vollständige Barrierefreiheit geben kann und muss, ist auch die umfassende Versorgungssicherheit innerhalb des Quartiers allein durch hauptamtliche Strukturen kaum möglich und auch nicht erstrebenswert. Vielmehr gilt es, die vorhandenen Selbsthilfepotenziale der Menschen zu stärken, informelle Netzwerke aufzubauen und $\mathrm{zu}$ erhalten, das Ehrenamt und bürgerschaftliches Engagement zu fördern. Damit ist die dritte zentrale Säule im
Bielefelder Modell angesprochen: die aktive Nachbarschaft, in der sich die Menschen entsprechend ihrer Fähigkeiten und Bedarfe füreinander einbringen.

Um dieses Ziel zu erreichen, bedarf es zunächst im engeren Bedeutungssinne des passenden Raumes. Daher ist wesentlicher Teil der Konzeptidee die Erstellung eines »Wohncafés", als Ort für ein privates Angebot von Bürgern für Bürger in der Nachbarschaft. Dieses wird seitens der Bielefelder Gemeinnützigen Wohnungsgesellschaft kostenfrei zur Verfügung gestellt und ist offen für alle Bürger im Quartier, unabhängig von Alter, Kultur oder Lebenslage. Insbesondere schafft es aber ein Angebot für Menschen, die aufgrund fehlender familiärer Netzwerke oder durch Krankheit oder Pflegebedürftigkeit einen besonderen Teilhabe- und Fürsorgebedarf haben. Tagesstrukturierende Ankerpunkte stellen die täglichen Mahlzeiten dar, die durch ehrenamtliche Köche zum Selbstkostenpreis zubereitet werden. Darüber hinaus bietet das Wohncafé Raum für weitere Aktivitäten, wie ge- 